\title{
Design of $4 \pi$ Directional Radiation Detector based on Compton Scattering Effect
}

\author{
Ghelman Max ${ }^{1,2}$, Kopeika Natan $^{2}$, Rotman Stenley $^{2}$, Edvabsky Tal $^{1}$, Vax Eran $^{1}$, Osovizky Alon ${ }^{1,3}$ \\ ${ }^{1}$ Electronics \& Control Laboratories, Nuclear Research Center, Negev, Israel \\ ${ }^{2}$ Department of Electrical and Computer Engineering, Ben-Gurion University of the Negev, Israel \\ ${ }^{3}$ Health Physics Instrumentation Department, Rotem Industries Ltd., Israel \\ maxghelman@gmail.com
}

\begin{abstract}
Obtaining directional information is required in many applications such as nuclear homeland security, contamination mapping after a nuclear incident and radiological events, or during the decontamination work. However, many directional radiation detectors are based on directional shielding, made of lead or tungsten collimators, introducing two main drawbacks. The first is the size and weight, making those detectors too heavy and irrelevant for utilization in handheld devices, drone mapping, or space applications. The second drawback is the limited field of view, which requires multiple detectors to cover the whole required field of view or machinery to rotate the narrow field of view detector. We propose a novel $4 \pi$ directional detector based on a segmented hollow cubic detector, which uses the Compton effect interactions with no heavy collimators. The symmetrical cubical design provides both higher efficiency and $4 \pi$ detection ability. Instead of traditional two types of detectors (scatterer and absorber) structure, we use the same type of detector, based on GAGG(Ce) scintillator coupled to silicon photomultiplier. Additional advantage of the proposed detector obtained by locating the photon sensors inside the detector, behind the scintillators, which improves the radiation hardness required for space applications. Furthermore, such arrangement flattens the temperature variation across the detector, providing better gain stability. The main advantage of the proposed detector is the ability of 4pi radiation detection for high energy gamma-rays without the use of heavy collimators.
\end{abstract}

Keywords - Silicon photomultiplier (SiPM), Compton scattering, directional detector, scintillator.

\section{INTRODUCTION}

$\mathrm{D}$ ETECTION of gamma ray sources is a challenging task in many applications, where monitoring and mapping of radiological and nuclear materials is required. Obtaining directional information is required for nuclear homeland security (HLS) and safety [1], where directional indication decreases source localization time by a factor of 2 to 10 [2]. Moreover, directional information is required for mapping of post-accident decontamination of nuclear incident such as Fukushima Daiichi and other radiological events [3[4].

Many directional radiation detectors are based on directional shielding [5[6] or coded mask [7] made of lead or tungsten collimators. Such concepts introduce three main drawbacks. The first is the size and weight that make those detectors too heavy and irrelevant for utilization in HLS handheld devices, drone mapping, or space applications. The second drawback is the small Field Of View (FOV). Proposed solutions that require multiple detectors to cover all the required FOV or machinery to rotate the limited FOV detector [8[9] increase size and weight even further. The third is the loss of events interacted in the collimator, which leads to lower of efficiency.

An efficient approach might be using mutual collimation detectors. As demonstrated by [10], 7 cylindrical 2" BGO scintillators provide sufficient shielding for high energies required for post-nuclear accident, such as $662 \mathrm{keV}$ emitted by ${ }^{137}$ Cs. Furthermore, [11] used the minimal possible three mutual collimated scintillates to provide directional information in two axes (horizontal). Opposite approach [12] used 90 to 340 $\mathrm{CsI}(\mathrm{Tl})$ scintillators in random and mutually shielding 3D structure to obtain $4 \pi$ directional detection. However, the efficiency drops significantly with increasing energy, practically limiting the directional efficiency up to about 100 $\mathrm{keV}$, which is insufficient for most post-nuclear accident isotopes. A different approach employs a 3D position-sensitive CZT Compton-based detector [13]. Such a CZT detector is successfully used for imaging, but the total efficiency is very low due to the small volume of about $2 \mathrm{~cm}^{3}$.

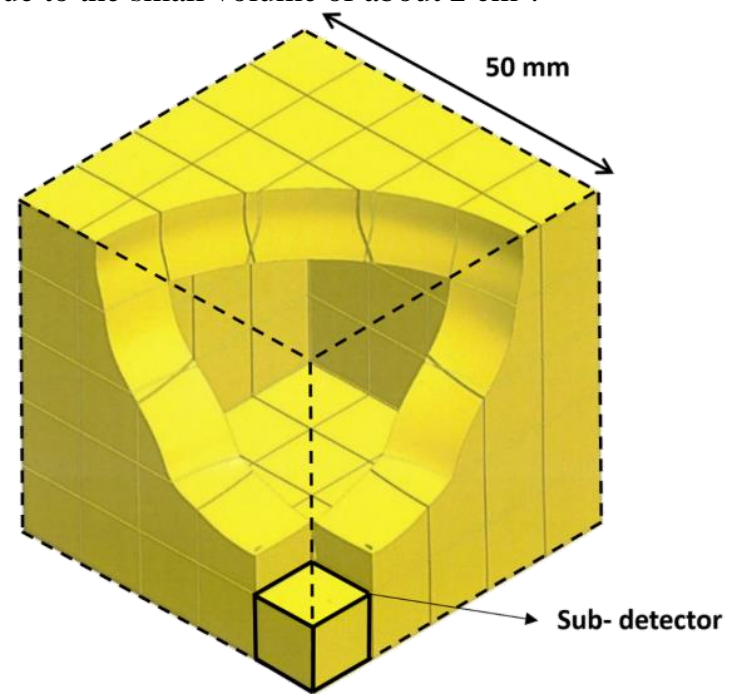

Fig. 1. The $4 \pi$ directional radiation detector configuration is based on a hollow cubical structure with a segmented outer shell. Each segment made of GAGG scintillator. The whole detector size is $50 \mathrm{~mm}$ X $50 \mathrm{~mm}$ X $50 \mathrm{~mm}$. 


\section{MEASUREMENT PRINCIPLE}

We propose a novel $4 \pi$ directional radiation detector based on Compton effect interactions. The basic segmented structure constructed of sub-detectors on a surface of a hollow cube is shown in Fig. 1. For lower energies, the photoelectric effect is dominant, and the approach of mutual shielding is preferred. At higher energies, instead of using various shielding methods for directional information, we make use of the Compton scattering events, see Fig. 2. Such events provide directional information in the form of scattered angle $\vartheta$ as defined in (1).

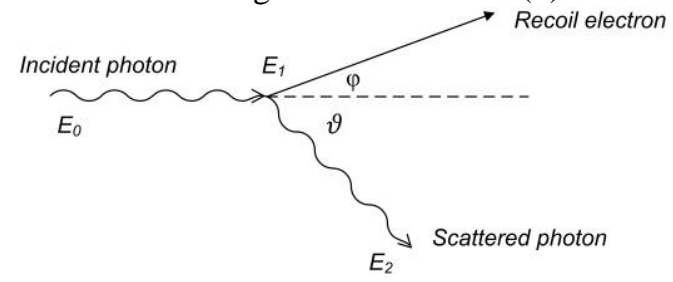

Fig. 2. Compton scattering of a gamma-ray photon and a recoil electron. The incident photon energy $E_{0}$, the electron energy $E_{l}$, and the angle $\vartheta$ dependence are defined by (1), scattered photon energy $E_{2}$ is not relevant.

$$
E_{1}=\frac{E_{0}}{1+\frac{h v}{m_{0} c^{2}}(1-\cos \vartheta)}
$$

Where, $m_{0} c^{2}$ is the electron rest energy, $E_{l}$ is the recoiled electron energy, and $E_{0}$ is the initial gamma photon energy, which is known for predefined isotopes. Measuring the scattered photon interaction location is required to establish the direction vector, see the dashed line in Fig. 3, which, in turn, is necessary to measure the angle $\vartheta$. Therefore, events with two or more interactions within the detector are required, see Fig. 3.

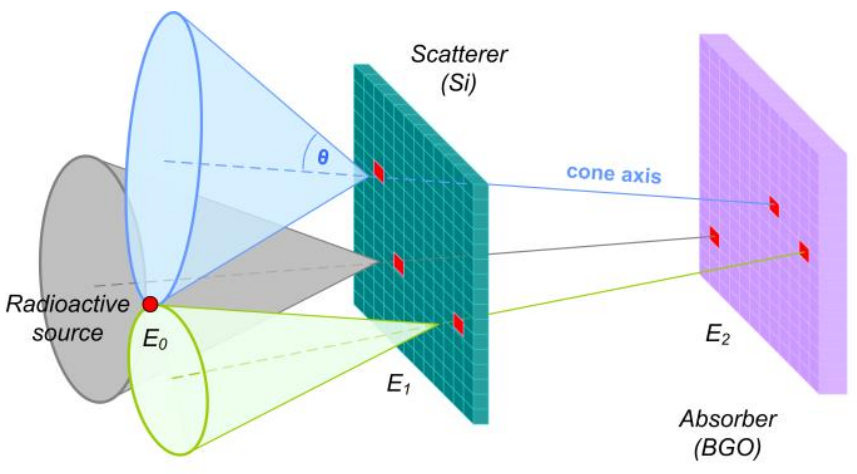

Fig. 3. Compton events with at least two simultaneous interactions within the detector. The conic probabilities define ellipses on a plane. The red dot in the intersection of those ellipses represents the radioactive source location.

For such interactions, when both the first Compton interaction, creating recoiled electron energy of energy $E_{l}$, and the second scattered gamma interaction locations were measured, the directional information embedded in the scattered angle $\vartheta$ is given by (2):

$$
\cos (\vartheta)=1-m_{0} c^{2}\left(\frac{1}{E_{0}-E_{1}}-\frac{1}{E_{0}}\right)
$$

When the isotope type is unknown, the scattered gamma energy must be measured in the absorber as well [14]. Equation (3) rearranges (2), so the missing isotope energy $E_{0}$ is replaced with the scattered photon energy $E_{2}$.

$$
\cos (\vartheta)=1-m_{0} c^{2}\left(\frac{1}{E_{2}}-\frac{1}{E_{1}+E_{2}}\right)
$$

Based on the interaction's locations of Compton-pairs events, we can back-project a probability cone of source locations. A series of events, generated by GAMOS software [15], was used to calculate a series of cones. These cones have one intersection point, which is the radioactive source location. Several algorithms were developed to overcome the uncertainties, noisy measurements, and limited resolution to converge the measured events into a single point source. The Maximum Likelihood Expectation Minimization ML-EM [16], provided the best results at this work.

\section{A. Material Selection}

A standard Compton camera is designed to have two types of materials. The scatterer material, which is selected for the first interaction, is a high energy-resolution detector with a low atomic number material, such as ${ }^{14} \mathrm{Si}$. Low atomic number decreases the photoelectric effect and maximizes the Compton effect probability. On the contrary, the absorber is selected to have maximal detection efficiency, without energy resolution requirements when using (2), such as BGO or LSO [17], or with energy resolution abilities when using (3) such as CdTe or CZT [18]. Maximal detection efficiency is achieved at high atomic number materials, which is opposite to the previously described qualities for the absorber. Nevertheless, we designed a symmetrical structure to provide a $4 \pi$ FOV; thus, we use the same material for both the absorber and the scatterer. Therefore, there is an inherent disagreement between some of the scintillation-materials properties requirements. Semiconductor materials such as Si or CZT, similarly, are not suitable for both types of detectors, due to the high noise at high temperatures of $50^{\circ} \mathrm{C}[19]$ required HLS applications.

Scintillators with low intrinsic radiation are preferable, since when such intrinsic gamma ray event occurs at the same measurement time-window with an external gamma event, they are erroneously considered as a Compton-pair.

The decay time property has a somewhat similar influence as intrinsic radiation. Longer decay times require a longer coincidence window, causing a higher probability to measure a false pair event. Scintillator energetic resolution has a direct influence on the measurement uncertainty of $\vartheta$, as calculated in [20], see (4). Hence, higher energy-resolution is advantageous for higher angular resolutions.

$$
\sigma(\vartheta)=\sigma\left(E_{1}\right) \cdot \frac{m_{0} c^{2}}{\sin (\vartheta) \cdot\left(E_{0}-E_{1}\right)^{2}}
$$

Scintillators in Table I with less than 4\% FWHM resolution $\left(\mathrm{LaBr}_{3}, \mathrm{SrI}_{2}\right.$, and $\left.\mathrm{CeBr}_{3}\right)$ are both hygroscopic and have intrinsic radiation, which makes them unsuitable. Scintillators with higher density, above $7 \mathrm{~g} / \mathrm{cm}^{3}$ (BGO and $\mathrm{CdWO}_{4}$ ), provide lower resolution, about $10 \%$. Considering all the scintillation properties and their influence on the performance with the considered scintillators in Table I, only the GAGG(Ce) scintillator provides fast decay time, high density, good FWHM resolution, and has no hygroscopic or intrinsic radiation deficiencies. The main drawback of the GAGG scintillator is that its maximal wavelength light yield, peaking at $530 \mathrm{~nm}$, is not compatible with the selected photon sensor, the Silicon Photo Multiplier (SiPM) discussed in the next chapter. The SiPM maximal quantum efficiency occurs at $420 \mathrm{~nm}$. 
TABLE I

SCINTILLATION CRYSTALS PROPERTIES

\begin{tabular}{|c|c|c|c|c|c|c|c|c|}
\hline Scintillator & CsI(Tl) & $\mathrm{Na}(\mathrm{Tl})$ & GAGG(Ce) & BGO & $\mathrm{CeBr}_{3}$ & $\mathrm{LaBr}_{3}$ & $\mathrm{SrI}_{2}(\mathrm{Eu})$ & $\mathrm{CdWO}_{4}$ \\
\hline Light yield (Photons / MeV) & 54000 & 38000 & 57000 & 8000 & 60000 & 63000 & 11500 & $15000^{\mathrm{b}}$ \\
\hline Resolution FWHM (\%) & $5.7^{\mathrm{a}}-7.1^{\mathrm{c}}$ & $6.8^{\mathrm{a}}-8.7^{\mathrm{c}}$ & $6.6^{\mathrm{a}}-10.5^{\mathrm{d}}$ & $9.5^{\mathrm{c}}-12^{\mathrm{h}}$ & $4.1^{\mathrm{e}}-4.4^{\mathrm{a}}$ & $2.9^{\mathrm{a}}-5.3^{\mathrm{c}}$ & $3.1^{\mathrm{g}}-4.4^{\mathrm{f}}$ & $8.5^{\mathrm{a}}-15.6^{\mathrm{c}}$ \\
\hline Hygroscopic & Slightly & Yes & No & No & Yes & Yes & Yes & No \\
\hline Density $\left(\mathrm{g} / \mathrm{cm}^{3}\right)$ & 4.51 & 3.67 & 6.63 & 7.13 & 5.1 & 5.2 & 4.6 & 7.9 \\
\hline Effective atomic number & 54 & 50 & $54 / 51$ fur & 72 & 46 & 45 & 50 & 64 \\
\hline Average time constant (ns) & 1000 & 250 & 90 & 300 & 20 & 25 & 1500 & 13000 \\
\hline Peak emission (nm) & 550 & 415 & 530 & 480 & 380 & 380 & 435 & 480 \\
\hline Intrinsic radiation & No & No & No & No & Slightly & Yes & Slightly & No \\
\hline
\end{tabular}

The relevant material properties of selected scintillators. ${ }^{a}$ - Measured in [21] with a Hamamatsu R6231-100 PMT; ${ }^{b}-$ by [22] with PMT; ${ }^{c}-$ by [23] with a Hamamatsu S11827-3344MG MPPC or XP 2020Q PMT; ${ }^{d}$ - by [24] with a SensL MicoFC SiPM; ${ }^{\mathrm{e}}$ - by [25] with Hamamatsu R6231 PMT; ${ }^{\mathrm{f}}$ - by [26] with a Hamamatsu TSV MPPC S12642-0404A-50; ${ }^{\text {- }}$ by [27] with a Hamamatsu R6231-100HA PMT; ${ }^{\text {h }}$ - by [28] with a SensL MicroFC-30035-SMT SiPM.

Such emission to absorption incompatibility lowers the number of measured photons and consequently decreases the measured resolution.

\section{B. Photon Sensor Selection}

Scintillation photons can be measured and amplified by a few types of photon sensors. The prime mandatory requirement for the segmented detector is compactness. Photomultiplier Tubes (PMT) are well based technology, with good noise performance at the required high temperatures but large and cumbersome, making the PMTs not suitable to be coupled to each of the small sub-detectors. The PIN diode and the Avalanche Photodiode (APD) are compact but undergo noise and gain degradation reaching $50^{\circ} \mathrm{C}$ [29]. The Silicon Photomultiplier (SiPM) is a compact sensor with good tiling ability that enables utilization of multiple sensors in proximity, without significant dead space between them. Furthermore, while the noise degradation with temperature is exponential, at $50^{\circ} \mathrm{C}$, noise levels are acceptable, degrading resolution by a factor of two, as measured with CsI(Tl) and ArrayJ-600035-64P SiPM [30].

The desired requirements for the light sensor include microphonic insensitivity. Sensors such as PIN diode that have no intrinsic gain are classically amplified by chargesensitive amplifiers. These amplifiers are sensitive to microphonic and piezoelectric noise. The SiPM provides a gain of millions of electrons for each measured photon. Such a high gain at the first stage is beneficial from electronic measurement SNR considerations and enables simpler and lower electronic amplification in the next stages. The bias voltage is only a few tens of volts instead of a few hundred volts for APDs and up to a few thousands of volts for PMTs. SiPMs are insensitive to electromagnetic interference [31, [32], which is an additional advantage over the classical PMT.

Nevertheless, there are three significant drawbacks of the SiPM technology that needs to be addressed. First, the radiation hardness of SiPMs is lower than PMTs, and they undergo a severe degradation in space, after a few years of operation at the international space station [33]. The other two drawbacks are gain variation with bias voltage and with temperature.

Bias variation requires stable and accurate voltage supply, with less than a few tens of $\mathrm{mV}$ variation over a few tens of volts bias voltage. Temperature-dependent gain stability can be achieved by stabilizing the temperature, which is not applicable for low power or handheld devices. A more practical approach is measuring the temperature $T$ and correcting the bias voltage, $\mathrm{V}_{\mathrm{B}}$, according to $(5,6)$.

$$
\begin{array}{r}
V_{B}=V_{B R}(T)+V_{O V}, \\
V_{B R}(T)=V_{B R}\left(T_{0}\right)+\beta \cdot\left(T-T_{0}\right),
\end{array}
$$

Where $V_{B R}$ is the temperature-dependent breakdown voltage, $T_{0}$ is a reference temperature, $\beta$ is the temperature coefficient, and $V_{O V}$ is the overvoltage. Since the gain of SiPM is $V_{O V}$ dependent, measuring $T$ and keeping $V_{O V}=V_{B}$ - $V_{B R}(T)$ constant will stabilize the gain.

\section{Geometrical Considerations}

To achieve a high geometrical resolution of radioactive source location, accuracy of scattered angle $\vartheta$ and the established directional vector is required. The scattered angle is primarily depended on the energy-resolution of the detector. The accuracy of the established directional vector, as defined by the dashed line in Fig. 3, requires high definition of the difference between the integration locations. Consequently, small detection segments and large distance between the first and the second interactions is desirable. Classically, this can be achieved by setting a few thin, low atomic number, pixelated scattering detectors in a distance from a high atomic number absorber, obtaining a high resolution in a narrow FOV [18] in one direction of the vector.

We propose using the same material for both the absorber and the scatterer to enable detection at both directions of the directional vector. A symmetrical cubic structure is formed by using three opposing pairs, providing a $4 \pi$ FOV directional detector. Furthermore, there is no requirement for the first and second events to occur in opposite pairs. Including the other four cube plains substantially increases the total detector efficiency.

The sub-detectors are located at the outer shell to obtain the maximal distance between interactions, producing a hollow cubical structure with a segmented scintillator surface (see Fig. 1). The whole detector size is $50 \mathrm{~mm} \mathrm{X} 50 \mathrm{~mm} \mathrm{X}$ $50 \mathrm{~mm}$. Each sub-detector is made of GAGG(Ce) scintillator coupled to an SiPM photon sensor. Each of the SiPMs needs to be measured thus requires multi-channel electronics $\left[34,[35]\right.$. The results in this work are for a cube of $10^{3}$ size 
requiring 488 of possible 512 channels.

The detector can be encapsulated with a rigid aluminum structure for the Compton scattering energy range without significant influence on the interaction efficiency. On the other hand, for the lower energies, where the photoelectric effect is predominant, adding structural material lowers the efficiency significantly. Using hygroscopic scintillators, especially for low-pressure space applications, would create additional encapsulation demands. These will reduce the efficiency even further. Hence, as mentioned before, they are not considered feasible for the proposed detector.

Furthermore, for the lower energy range used in directional radiation detectors, even thin PCBs are of much consideration for loss of efficiency [12]. The proposed hollow cube configuration's outer layer is rigid by itself, made of GAGG scintillators, so there is no need for additional significant structure. The PCBs and the SiPM sensors are located inside the cube volume, so there is no shielding at the front of the scintillators.

Furthermore, positioning the SiPMs inside the detector provides a significant gain stability advantage. Since SiPM gain is temperature-dependent, the temperature is measured, and the bias voltage is adjusted according to (5) for gain stability.

An additional advantage achieved by locating the SiPMs inside is enhanced radiation hardness. As stated in the properties of the photon sensors in Table I and demonstrated in [33[36] when irradiated, SiPMs experience a large increase in dark current, increasing power consumption and noise, limiting the detection of low energy events. Hence, shielding the SiPMs from external radiation with a dense scintillator could provide prolonged lifetime of the detector.

\section{RESULTS}

The presented results are for the $10^{3}$, cube size, using a GAGG(Ce) based $5 \mathrm{~mm}$ X $5 \mathrm{~mm}$ X 5mm sub-detectors with $7 \%$ FWHM energy resolution. Results of one side projection are shown on the right side of Fig. 4 (colored estimated distribution on the black background). The detector is located at the origin (blue), with the directional vectors (green) shown inside the detector. The red arrow represents the location and direction of the estimated radioactive source.

The performance of the proposed detector was evaluated for the $662 \mathrm{keV}$ of the ${ }^{137} \mathrm{Cs}$ isotope. The final estimation is based on five iterations of ML-EM, reaching an estimation accuracy of $\sigma(\vartheta)=2^{\circ}$.

\section{CONCLUSIONS}

This work presents a novel $4 \pi$ directional radiation detector. We designed a detector based on a Compton scattering effect as a hollow cube-shaped segmented scintillator. We have demonstrated that without using collimators, a $4 \pi$ directional detection of high-energy isotopes is feasible. Typically, directional detection for nuclear incidents requires not only high gamma energy measurement capability, but also composed of a few radioactive sources.

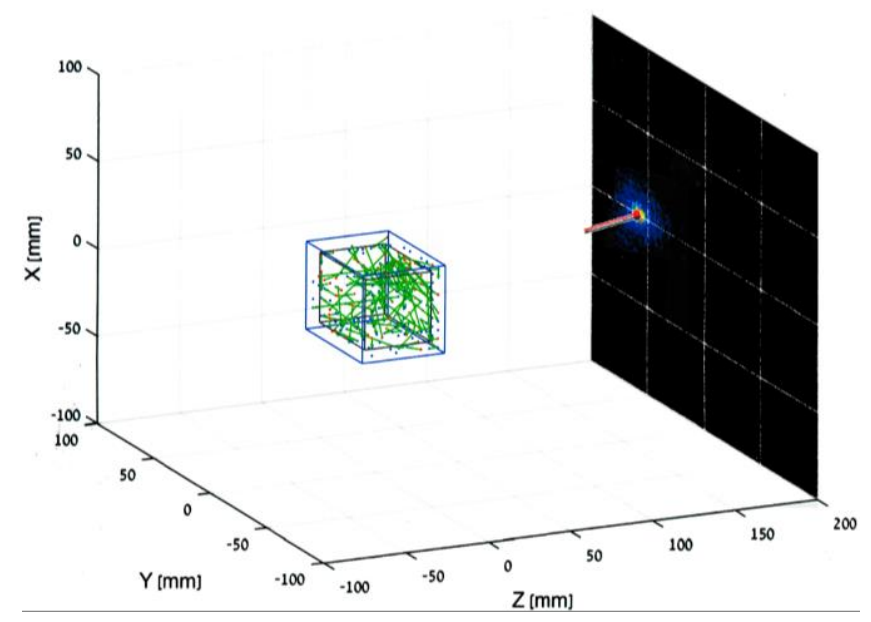

Fig. 4. Estimation of $662 \mathrm{keV}{ }^{137} \mathrm{Cs}$ isotope. The detector located at the origin (blue), with the directional vectors (green) shown inside the detector. The red arrow represents the location and direction of the estimated radioactive source. Colored estimation distribution is shown on the black background.

While using the same type of scintillators for both the absorber and the scatterer detectors is not optimal, such an approach enables us to double the measurable field of view. Moreover, the selected GAGG scintillator is sufficient to obtain a high directional accuracy of about $2^{\circ}$.

Utilizing any one of the six cube planes as scatterer detector, while using the other five as absorbers, provides $4 \pi$ FOV and increases the efficiency, since more Compton pairs are available in comparison to the conventional two planes design (one scatterer and one absorber), which limits the direction of arrival of photons.

The GAGG(Ce) scintillator coupled to an SiPM photon sensor as a sub-detector segment provides the most suitable overall properties for the proposed hollow cube configuration detector.

Hollow cube configuration with the SiPMs located inside provides the ability to stabilize the temperature and thus stabilize the gain while protecting the delicate photon sensors from radiation, providing an extended lifetime.

\section{REFERENCES}

[1] “AccuRad ${ }^{\mathrm{TM}}$ PRD, Personal Radiation Detector", [Online]. Available: https://www.mirion.com/products/accurad-prd-personalradiation-detector. Accessed on: June 05, 2021.

[2] C. L. Larsson, T. Jones, "Testing a new directional gamma survey meter", Defense R\&D Canada - Ottawa, 2012, [Online]. Available: https://cradpdf.drdc-rddc.gc.ca/PDFS/unc240/p803606_A1b.pdf. Accessed on: June 05, 2021.

[3] Y. Sato, Y. Tanifuji, Y. Terasaka, H. Usami, M. Kaburagi, K. Kawabata, et al., "Radiation imaging using a compact Compton camera inside the Fukushima Daiichi Nuclear Power Station building", Journal of Nuclear Science and Technology, 2018, Vol. 55 No. 9, 965-970, DOI:10.1080/00223131.2018.1473171.

[4] J. Jiang, K. Shimazoe, Y. Nakamura, H. Takahashi, Y. Shikaze, Y. Nishizawa, et al., "A prototype of aerial radiation monitoring system using an unmanned helicopter mounting a GAGG scintillator Compton camera", Journal of Nuclear Science and Technology, 53:7, 1067-1075, DOI:10.1080/00223131.2015.1089796.

[5] V. Batiy, O. Stoyanov D. Fedorchenko, S. Prohoretz, M. Khazhmuradov, "Mathematical modeling to support gamma 
radiation angular distribution measurements", Waste Management Symposium, Tucson, AZ (United States), January 2007; https://www.researchgate.net/publication/237505414.

[6] K. Kojima, T. Nakamori, D. Nemoto, S. Gunji, H. Sato, S. Ito, S. Kato, M. Yoshino, Y. Usuki, J. Kataoka, "Development of GammaRay Detector Sensitive to Source Directions using GAGG(Ce) Scintillators and MPPCs", IEEE- NSS October 2016, DOI: 10.1109/NSSMIC.2016.8069923.

[7] O. Gala, M Gmara , O. P. Ivanovb , F. Laine, F. Lamadiec , C. Le Goallerc, et al., "Development of a portable gamma camera with coded aperture", 563(1):233-237, NIM A 563 (2006) 233-237 DOI:10.1016/j.nima.2006.01.119.

[8] O. Gala Bryan V. Egner, Darren E. Holland, Larry W. Burggraf, James E. Bevins, "Development of a modular mixed-radiation directional rotating scatter mask detection system". 987, NIM A, 2021, 164820, DOI:10.1016/j.nima.2020.164820.

[9] G. Cao, B. Liu, H. Gong, H. Yu and G. Wang, "A StationarySources and Rotating-Detectors Computed Tomography Architecture for Higher Temporal Resolution and Lower Radiation Dose", IEEE Access, vol. 2, pp. 1263-1271, 2014, DOI: 10.1109/ACCESS.2014.2363367.

[10] A. Pernick, I. Orion, D. Ilzycer, H Zafrir, “Applications of a SelfCollimating BGO Detector System to Radiological Emergency Response", Health Phys. 1997 Jan; 72(1):136-40. DOI:10.1097/00004032-199701000-00019.

[11] Y. Shirakawa, T. Yamano and Y. Kobayashi, "Remote sensing of nuclear accidents using a direction-finding detector," 2009 35th Annual Conference of IEEE Industrial Electronics, 2009, pp. 1917 1922, DOI:10.1109/IECON.2009.5414850.

[12] R. Rahin, L. Moleri, A. Vdovin, A. Feigenboim, S. Margolin, S. Tarem, et al., "GALI: a gamma ray burst localizing instrument", Proc. SPIE 11444, Space Telescopes and Instrumentation 2020: Ultraviolet to Gamma Ray, 114446E, 2020, DOI:10.1117/12.2576126.

[13] Dan Xu, Z. He, C. E. Lehner, F. Zhang, “ $4 \pi$ Compton imaging with single 3D position sensitive CdZnTe detector", 2004, Proceedings Volume 5540, Hard X-Ray and Gamma-Ray Detector Physics VI, DOI: https://doi.org/10.1117/12.563905.

[14] Daniel W. Mundy and Michael G. Herman, "Uncertainty analysis of a Compton camera imaging system for radiation therapy dose reconstruction", 2010, American Association of Physicists in Medicine, DOI: 10.1118/1.3399777.

[15] P. Arce. P. Rato, M. Canadas, and J. I. Lagares, "GAMOS: A Geant4-based easy and flexible framework for nuclear medicine applications," IEEE Nuclear Science Symposium Conference Record, pp. 3162-3168, 2008.

[16] S. J. Wilderman, N.H. Clinthorne, J.A. Fessler, W. L. Rogers, "ListMode Maximum Likelihood Reconstruction of Compton Scatter Camera Images in Nuclear Medicine", 1998, IEEE Nuclear Science Symposium and Medical Imaging Conference, DOI: 10.1109/NSSMIC.1998.773871.

[17] F. Hueso-Gonzalez, A. K. Biegun, P. Dendooven, W. Enghardt, F. Fiedler, C. Golnik, at. al., "List-Mode Maximum Likelihood Reconstruction of Compton Scatter Camera Images in Nuclear Medicine", 2015 JINST 10 P09015, DOI: http://dx.doi.org/10.1088/1748-0221/10/09/P09015.

[18] T. Takahashi, M. Kokubun, K. Mitsuda, R. L. Kelley, T. Ohashi, F. Aharonian, et. al., "The ASTRO-H X-ray Astronomy Satellite", 2018, J. of Astronomical Telescopes, Instruments, and Systems, 4(2), 021402, DOI:https://doi.org/10.1117/1.JATIS.4.2.021402.

[19] S. H. Park, J. H. Ha, J. H. Lee, H. S. Kim, Y. H. Cho, et. al. "Effect of Temperature on the Performance of a CZT Radiation Detector", Journal of the Korean Physical Society, Vol. 56, No. 4, April 2010, pp. $1079 \sim 1082$.

[20] E. Munoz1, J. Barrio1, A. Etxebeste, P. G. Ortega, C. Lacasta, J. F. Oliver, et. al.," Performance evaluation of MACACO: a multilayer Compton camera", Phys. Med. Biol. 62 (2017) 7321-7341, DOI:https://doi.org/10.1088/1361-6560/aa8070.

[21] P. Sibczynski, A. Broslawski, A. Gojska, V. Kiptily, S. Korolczuk, R. Kwiatkowski, et.al. "Characterization of some modern scintillators recommended for use on large fusion facilities in g-ray spectroscopy and tomographic measurements of g-emission profiles", Jun. 2017 Nukleonika 62(3):223 DOI:10.1515/nuka-2017$\underline{0032 .}$.
[22] L. Nagornaya, S. Burachas, Y. Vostretsov, V. Martynov, V. Ryzhikov, "Studies of ways to reduce defects in CdWO4single crystals", Journal of Crystal Growth 198/199 (1999) 877-880, DOI: 10.1016/S0022-0248(98)01236-6.

[23] M. Grodzicka, M. Moszynski, T. Szczesniak, M. Szawlowskia, J. Baszak, "Characterization of $4 \times 4 \mathrm{ch}$ MPPC array in scintillation spectrometry", 2012 IEEE Nuclear Science Symposium and Medical Imaging Conference Record (NSS/MIC), DOI:10.1109/NSSMIC.2012.6551118.

[24] B. Seitz, N. Campos Rivera, and A. G. Stewart, "Energy Resolution and Temperature Dependence of Ce:GAGG Coupled to $3 \mathrm{~mm} \times 3$ mm Silicon Photomultipliers", IEEE TRANSACTIONS ON NUCLEAR SCIENCE, VOL. 63, NO. 2, APRIL 2016 DOI: 10.1109/TNS.2016.2535235.

[25] F.G.A.Quaratiab, P.Dorenbosa, J. van der Biezenc, A Owensc, M. SelledL, P. Schotanusf, "Scintillation and detection characteristics of high-sensitivity CeBr3 gamma-ray spectrometers", 2013 NIM A, 729 596-604, DOI: 10.1016/j.nima.2013.08.005.

[26] K. Shimazoe, , A. Koyama, H. Takahashi, S. Sakuragi, Y. Yamasaki, "Fabrication and characterization of rectangular strontium iodide scintillator coupled to TSV-MPPC array", NIM A 845 503-506, 2017, DOI: 10.1016/j.nima.2016.06.077

[27] M. Takabe a,n, A. Kishimoto a , J. Kataoka a, S. Sakuragi b, Y. Yamasaki, "Performance evaluation of newly developed SrI2(Eu) scintillator", NIM A 831 260-264, 2016, DOI: $10.1016 /$ j.nima.2016.04.043.

[28] A. J. González, F. Sanchez, S. Majewski, A. Aguilar, A. GonzálezMontoro, et. al., "Performance of large BGO arrays coupled to SiPM photosensors - Continued study", 2015 IEEE Nuclear Science Symposium and Medical Imaging Conference (NSS/MIC), 2015, pp. 1-4, DOI: 10.1109/NSSMIC.2015.7582017.

[29] Si APD, MPPC, Handbook chapter 3, E03, [Online]. Available: https://www.hamamatsu.com/resources/pdf/ssd/e03_handbook_si_a pd mppc.pdf. Accessed 14.07.2021.

[30] J. Valencia, B. Godfrey, M. Hamel, B. Maestas, E. Padilla, "Evaluation of a Silicon Photomultiplier Array as a Photomultiplier Tube Replacement", 2019, SAND2019-6405C. Sandia National Laboratories2, Albuquerque, NM, USA. [Online]. Available: https://www.osti.gov/servlets/purl/1640659. Accessed 14.07.2021.

[31] M. Ren, J. Zhou, B. Song, C. Zhang, M. Dong, R. Albarracin, "Towards Optical Partial Discharge Detection with Micro Silicon Photomultipliers", Sensors 2017, 17(11), 2595 DOI: https://doi.org/10.3390/s17112595.

[32] R. Hawkes, A. Lucas, J. Stevick, G. Llosa, S. Marcatili, C. Piemonte, "Silicon photomultiplier performance tests in magnetic resonance pulsed fields", 2007, IEEE Nuclear Science Symposium Conference Record, DOI:10.1109/NSSMIC.2007.4436860.

[33] A. Ulyanov, D. Murphy, J. Mangan, V. Gupta, W. Hajdas, at. al., "Radiation damage study of SensL J-series silicon photomultipliers using 101.4 MeV protons", NIM A, 976 (2020) 164203, DOI:https://doi.org/10.1016/j.nima.2020.164203.

[34] D. Impiombato, S. Giarrusso, T. Mineo, , O. Catalano, C. Gargano, G. La Rosa, et. al., "Characterization and performance of the ASIC (CITIROC) front-end of the ASTRI camera", NIM A, 794 (2015) 185-192, DOI:10.1016/j.nima.2015.05.028.

[35] V. Nadig, D. Schug, B. Weissler, V. Schulz, "Evaluation of the PETsys TOFPET2 ASIC in multi-channel coincidence experiments", EJNMMI Physics volume 8, Article number: 30 (2021), DOI:https://doi.org/10.1186/s40658-021-00370-x.

[36] K. Lacombe, J. Knodlseder, B. Houret, T. Gimenez, J. F. Olive, P. Ramon, "Characterization of silicon photomultipliers for new highenergy spacetelescopes", NIM A, 912 (2018) 144-148, DOI:https://doi.org/10.1016/j.nima.2017.11.005. 